\title{
Exploring the photoleakage current and photoinduced negative bias instability in amorphous InGaZnO thin-film transistors with various active layer thicknesses
}

\author{
Dapeng Wang ${ }^{* 1}$ and Mamoru Furuta ${ }^{* 2,3}$
}

\author{
Full Research Paper \\ Address: \\ ${ }^{1}$ Key Laboratory of Applied Surface and Colloid Chemistry, Ministry of \\ Education; Shaanxi Key Laboratory for Advanced Energy Devices; \\ Shaanxi Engineering Lab for Advanced Energy Technology, School of \\ Materials Science and Engineering, Shaanxi Normal University, Xi'an \\ 710119, China, ${ }^{2}$ School of Environmental Science and Engineering, \\ Kochi University of Technology, Kami, Kochi 782-8502, Japan and \\ ${ }^{3}$ Center for Nanotechnology in Research Institute, Kochi University of \\ Technology, Kami, Kochi 782-8502, Japan

\section{Email:} \\ Dapeng Wang* - dpwang@snnu.edu.cn; Mamoru Furuta* - \\ furuta.mamoru@kochi-tech.ac.jp \\ * Corresponding author \\ Keywords: \\ active layer thickness; gate bias; illumination stress; InGaZnO; \\ photoleakage current; thin-film transistors
}

Beilstein J. Nanotechnol. 2018, 9, 2573-2580.

doi:10.3762/bjnano.9.239

Received: 09 May 2018

Accepted: 07 September 2018

Published: 26 September 2018

Associate Editor: E. Meyer

() 2018 Wang and Furuta; licensee Beilstein-Institut. License and terms: see end of document.

\begin{abstract}
The photoleakage current and the negative bias and illumination stress (NBIS)-induced instability in amorphous InGaZnO thin-film transistors (a-IGZO TFTs) with various active layer thicknesses $\left(T_{\text {IGZO }}\right)$ were investigated. The photoleakage current was found to gradually increase in a-IGZO TFTs irrespective of the $T_{\text {IGZO }}$ when the photon energy of visible light irradiation exceeded $\approx 2.7 \mathrm{eV}$. Furthermore, the influence of the $T_{\text {IGZO }}$ on NBIS-induced instability in a-IGZO TFTs was explored by the combination of current-voltage measurements in double-sweeping $V_{\mathrm{GS}}$ mode and capacitance-voltage measurements. The NBIS-induced hysteresis was quantitatively analyzed using a positive gate pulse mode. When the $T_{\mathrm{IGZO}}$ was close to the Debye length, the trapped electrons at the etch-stopper/IGZO interface, the trapped holes at the IGZO/gate insulator interface, and the generation of donor-like states in an a-IGZO layer were especially prominent during NBIS.
\end{abstract}

\section{Introduction}

Over the last decade, the amorphous oxide-based semiconductor thin-film transistors (AOS TFTs) have attracted global attention for use in advanced display technologies due to their outstanding properties such as high electron mobility, good transparency to visible light, and low process temperature with good uniformity [1-4]. Among the numerous AOS materials, indium gallium zinc oxide (IGZO) is one of the most promising candidates used as the active layer because of its excellent electrical and optical properties [5-8]. Although the band gap of IGZO $(\approx 3.1 \mathrm{eV})$ is higher than the photon energy of visible light, pho- 
toinduced leakage current under visible-light irradiation can be detected in the oxide-based TFTs $[9,10]$. This is due to the fact that the electrons are excited from the trapped states existing near the valence band $\left(E_{\mathrm{V}}\right)$. In addition, the a-IGZO TFTs inevitably suffer electrical and optical stresses during practical operation conditions, especially for the negative bias and illumination stress (NBIS) tests [11-16], which leads to device instability and restricts the development of oxide TFTs for commercial products.

In our previous study, a double-sweeping $V_{\mathrm{GS}}$ mode was proposed to investigate the origin of NBIS-induced hysteresis of a-IGZO TFTs [17]. A promising method to suppress NBIS degradation was also considered by applying a large negative $V_{\mathrm{DS}}$ bias of $V_{\mathrm{DS}}<V_{\mathrm{GS}}$ during NBIS [18]. These studies imply that the fabrication parameters for the active layer should be well taken into account to improve the reliability of oxide TFTs. The active layer thickness is a key parameter to modify the performance of a-IGZO TFTs. Some works have highlighted that the electrical properties of the device (for both the initial and after stress conditions) such as threshold voltage, on/off ratio, and field effect mobility, can be effectively adjusted by controlling the active layer thickness [19-23]. Up to now, the impact of the active layer thickness ( $T_{\text {IGZO }}$ ) on the photoleakage current and NBIS-induced instability in a-IGZO TFTs has been rarely reported. The NBIS-induced degradation of a-IGZO TFTs with various active layer thicknesses has also rarely been discussed.

In this study, a-IGZO films with various active layer thicknesses were prepared by magnetron sputtering. The initial electrical properties and the photoleakage current of a-IGZO TFTs with various active layer thicknesses were investigated. The subthreshold value slightly increased while the threshold voltage $\left(V_{\text {th }}\right)$ and mobility $(\mu)$ decreased with increasing $T_{\text {IGZO }}$ The photoleakage current increased in all TFTs when the wavelength of visible-light irradiation was shorter than $460 \mathrm{~nm}$.
Moreover, the photoleakage current increased with an increase in the $T_{\mathrm{IGZO}}$. Furthermore, the impact of the active layer thickness on the NBIS-induced instability in a-IGZO TFTs was explored by combining the current-voltage $(I-V)$ measurements in double-sweeping $V_{\mathrm{GS}}$ mode and capacitance-voltage $(C-V)$ measurements. The NBIS-induced hysteresis was quantitatively analyzed using a positive gate pulse mode. The $I-V$ and $C-V$ results revealed that the trapped holes at the etch-stopper/ IGZO interface, the trapped holes at the IGZO/gate insulator interface, and the generation of donor-like states in a-IGZO layer were particularly prominent after NBIS tests when the active layer thickness was close to the Debye length.

\section{Experimental}

A schematic cross-sectional view of a bottom-gate a-IGZO TFT is shown in Figure 1a. The detailed fabrication procedure for the a-IGZO TFT was described in our previous publication [24]. After the fabrications of a chromium $(\mathrm{Cr})$ gate electrode and a $\mathrm{SiO}_{x}$ gate insulator $(150 \mathrm{~nm})$, the a-IGZO layer with thicknesses of $25,45,75$, and $100 \mathrm{~nm}$ respectively were deposited at $160{ }^{\circ} \mathrm{C}$ from a sintered IGZO ceramic target by DC magnetron sputtering with a mixed gas of $\mathrm{Ar} / \mathrm{O}_{2}=29.4 / 0.6 \mathrm{sccm}$ at a deposition pressure of $1 \mathrm{~Pa}$. After patterning of the IGZO films as the active channel, a $\mathrm{SiO}_{x}$ etch-stopper $(200 \mathrm{~nm})$, source and drain electrodes, and a $200 \mathrm{~nm}$-thick $\mathrm{SiO}_{x}$ passivation layer were sequentially formed. Following the preparation of a-IGZO TFTs, all devices were annealed in $\mathrm{N}_{2}$ environment at $350{ }^{\circ} \mathrm{C}$ for $1 \mathrm{~h}$ before the electrical measurements. The channel width $(W)$ and length $(L)$ of the IGZO TFTs were 50 and $20 \mu \mathrm{m}$, respectively. All of the $I-V$ characteristics were measured using an Agilent $4156 \mathrm{C}$ precision semiconductor parameter analyzer.

For the photoleakage current test, monochromatic light irradiation was supplied by a Xe lamp with a band pass filter (FWHM of $10 \mathrm{~nm}$ ) at an intensity of $0.2 \mathrm{~mW} / \mathrm{cm}^{2}$. The wavelength of the light was in the range of $400-530 \mathrm{~nm}$ and was introduced to the (a)

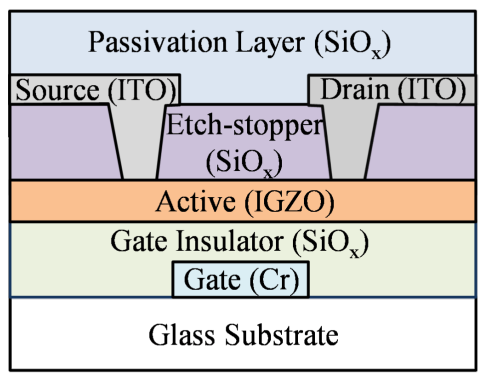

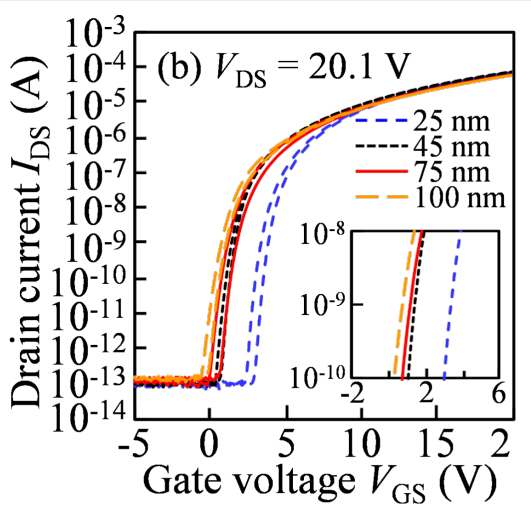

Figure 1: (a) Schematic cross-sectional view and (b) the initial transfer characteristics of a-IGZO TFTs with various active layer thicknesses ( $T_{I G Z O}$ ) measured at $V_{\mathrm{DS}}=20.1 \mathrm{~V}$. 
back-channel side of a-IGZO TFTs. For the NBIS test, blue light with a wavelength of $460 \mathrm{~nm}$ and a gate voltage $\left(V_{\mathrm{GS}}\right)$ of $-30 \mathrm{~V}$ was simultaneously applied to all devices during the stress test. The NBIS was interrupted briefly when the transfer characteristics were measured in double-sweeping $V_{\mathrm{GS}}$ mode in darkness at $V_{\mathrm{DS}}=0.1 \mathrm{~V}$, and then NBIS was reapplied up to a stress time of $10^{4} \mathrm{~s}$. In terms of the double-sweeping $V_{\mathrm{GS}}$ mode, the transfer characteristics were measured with $V_{\mathrm{GS}}=-10-20 \mathrm{~V}$ (denoted hereafter as forward measurement), and then scanned instantly back to $-10 \mathrm{~V}$ (denoted hereafter as reverse measurement). The $C-V$ measurements for the channel capacitance were measured at $1 \mathrm{kHz}$ and an AC level of $100 \mathrm{mV}$. All of the measurements were carried out at room temperature in ambient air.

\section{Results and Discussion}

The initial transfer characteristics $\left(I_{\mathrm{DS}}-V_{\mathrm{GS}}\right)$ of a-IGZO TFTs with various active layer thicknesses $\left(T_{\mathrm{IGZO}}\right)$ measured at $V_{\mathrm{DS}}=20.1 \mathrm{~V}$ are shown in Figure 1b. Table 1 summarizes the

$\begin{aligned} & \text { Table 1: The initial electrical properties of a-IGZO TFTs with various } \\
& \text { active layer thicknesses }\left(T_{\text {IGZO }}\right)\end{aligned}$
\begin{tabular}{lllll} 
Thickness, $T_{\text {IGZO }}(\mathrm{nm})$ & 25 & 45 & 75 & 100 \\
\hline$\mu_{\text {sat }}\left(\mathrm{cm}^{2} \cdot \mathrm{V}^{-1} \cdot \mathrm{s}^{-1}\right)$ & 13.61 & 13.47 & 12.43 & 12.20 \\
$V_{\mathrm{GS}}$ at $I_{\mathrm{DS}}=1 \mathrm{nA}(\mathrm{V})$ & 3.35 & 1.24 & 1.16 & 0.74 \\
hysteresis $\Delta V_{\mathrm{H}}(\mathrm{V})$ & 0.47 & 0.42 & 0.35 & 0.30 \\
subthreshold swing $(\mathrm{mV} /$ dec. $)$ & 264 & 273 & 316 & 352 \\
trap density $\left(10^{11} \mathrm{~cm}^{-2}\right)$ & 5.06 & 5.29 & 6.35 & 7.24
\end{tabular}

electrical properties, such as field effect mobility in the saturation region $\left(\mu_{\mathrm{sat}}\right)$, threshold voltage $V_{\text {th }}\left(V_{\mathrm{GS}}\right.$ at $I_{\mathrm{DS}}$ of $\left.1 \mathrm{nA}\right)$, hysteresis of the transfer curves (the difference of $V_{\mathrm{GS}}$ at $I_{\mathrm{DS}}$ of $1 \mathrm{pA}$ between the forward and reverse sweeps), subthreshold swing ( $\left.\mathrm{SS}=\mathrm{d} V_{\mathrm{GS}} / \mathrm{dlog}_{10}\left(I_{\mathrm{DS}}\right)\right)$, and the maximum area density of state $\left(N_{\mathrm{t}}\right)$.

Compared to a previous publication [25], the electrical properties of a-IGZO TFTs with various $T_{\text {IGZO }}$ exhibit the identical tendency. It is suggested that the devices exhibit great repeatability for the same kind of material under the same fabrication process. In addition, the $V_{\text {th }}$ results demonstrate that the free carrier numbers in the bulk of the active layer are gradually increased with increasing $T_{\mathrm{IGZO}}$. Moreover, since the fabrication condition for the IGZO films are exactly identical, except the deposition duration, the results suggest that the variation in the SS value mainly originates from the density of defect states in the active layer. Correspondingly, the obtained results indicate that the increase in the $N_{\mathrm{t}}$ majorly stems from the increase of the IGZO bulk traps because of the identical a-IGZO/GI interfaces.

Figure $2 \mathrm{a}-\mathrm{d}$ shows the variation in the transfer characteristics of a-IGZO TFTs with various $T_{\text {IGZO }}$ under monochromatic light irradiation at the excitation wavelengths of 400, 430, 460, 490, and $530 \mathrm{~nm}$. The transfer characteristics measured in the dark are also shown as a reference. The transfer characteristics of all devices are measured with a $V_{\mathrm{DS}}$ of $10.1 \mathrm{~V}$ and a $V_{\mathrm{GS}}$ scanned from the on-to-off direction. It is found that the photoleakage

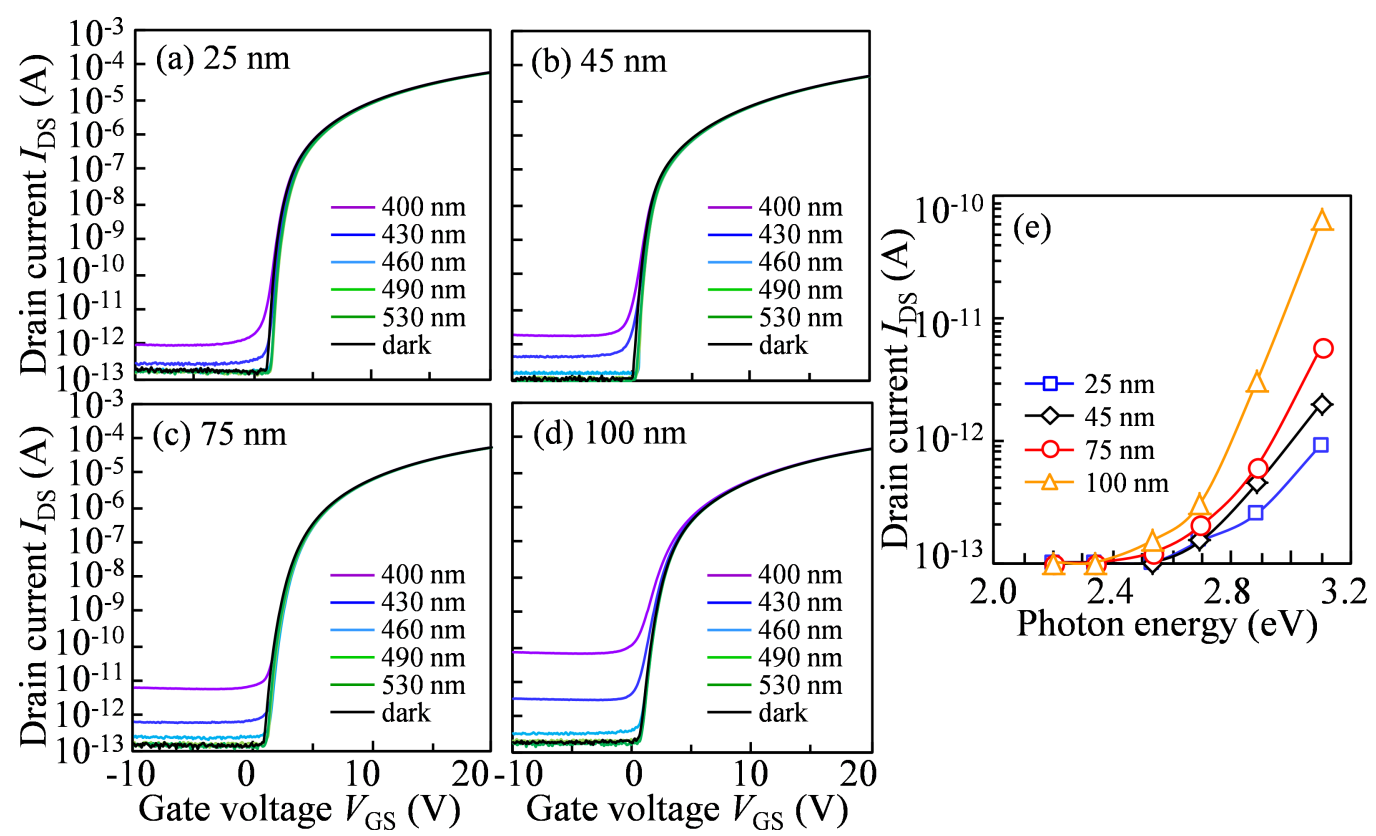

Figure 2: Variation in the transfer characteristics of a-IGZO TFTs with (a) $T_{1 G Z O}=25$, (b) 45 , (c) 75 , and (d) $100 \mathrm{~nm}$ under monochromatic light irradiation, and $(\mathrm{e})$ photoleakage current $\left(V_{\mathrm{GS}}=-10 \mathrm{~V}\right.$ and $\left.\mathrm{V}_{\mathrm{DS}}=10 \mathrm{~V}\right)$ of a-IGZO TFTs with various thicknesses as a function of photon energy of the incident light. 
current increases in all TFTs when the irradiation wavelength is shorter than $460 \mathrm{~nm}$. In addition, the photoleakage current increases with increasing $T_{\text {IGZO }}$. Figure $2 \mathrm{e}$ exhibits the photoleakage current of a-IGZO TFTs with various $T_{\mathrm{IGZO}}$ as a function of the photon energy of incident light. When the photon energy exceeds $\approx 2.7 \mathrm{eV}(460 \mathrm{~nm})$, the photoleakage current starts to increase and increases gradually with increasing photon energy. Note that the photoleakage current increases dramatically in the photon energy range of $>2.7 \mathrm{eV}$ for the TFT with the thicker $T_{\text {IGZO }}$. These results indicate that the electrons are excited from the trapped states existing near the valence band $\left(E_{\mathrm{V}}\right)$ to the conduction band $\left(E_{\mathrm{C}}\right)$ even though the photon energy is smaller than the band gap of IGZO. In terms of the a-IGZO material, the high-density electron traps exist at $\left(E_{\mathrm{C}}-E\right)$ of over $2.7 \mathrm{eV}$ [9], which affect the photoleakage current of a-IGZO TFTs. The total amount of trapped electrons increase with an increase in the $T_{\text {IGZO }}$. The oxygen-related defects, such as oxygen vacancies $\left(\mathrm{V}_{\mathrm{O}}\right)$, may be the origin of high-density electron traps near the $E_{\mathrm{V}}$ in a-IGZO TFTs, which occupy the region near the valence band maximum with an energy width of $\approx 1.5 \mathrm{eV}$ $[26,27]$.

Figure $3 \mathrm{a}-\mathrm{d}$ shows the variation in the transfer characteristics of a-IGZO TFTs with various $T_{\text {IGZO }}$ under NBIS for the forward measurements. It is found that for the NBIS duration of $1000 \mathrm{~s}$, the transfer curves of all TFTs shift in the negative $V_{\mathrm{GS}}$ direction without SS degradation. When the NBIS duration exceeds $1000 \mathrm{~s}$, a shift in the positive $V_{\mathrm{GS}}$ direction as well as the appearance of a hump with SS degradation in the transfer curves is observed, and this phenomenon gradually increases with increasing NBIS duration. It is noted that the phenomenon, combined the positive shift and the hump effect, is weakened as the $T_{\text {IGZO }}$ increases. For the reverse measurements, as shown in Figure $3 \mathrm{e}-\mathrm{h}$, the transfer curves of all TFTs shift parallel in the positive $V_{\mathrm{GS}}$ direction without SS degradation during the NBIS duration of $>1000$ s. Noticeably, the abnormal phenomenon of hump appearance observed in the forward measurements is hardly observed in the reverse measurements. The positive shift
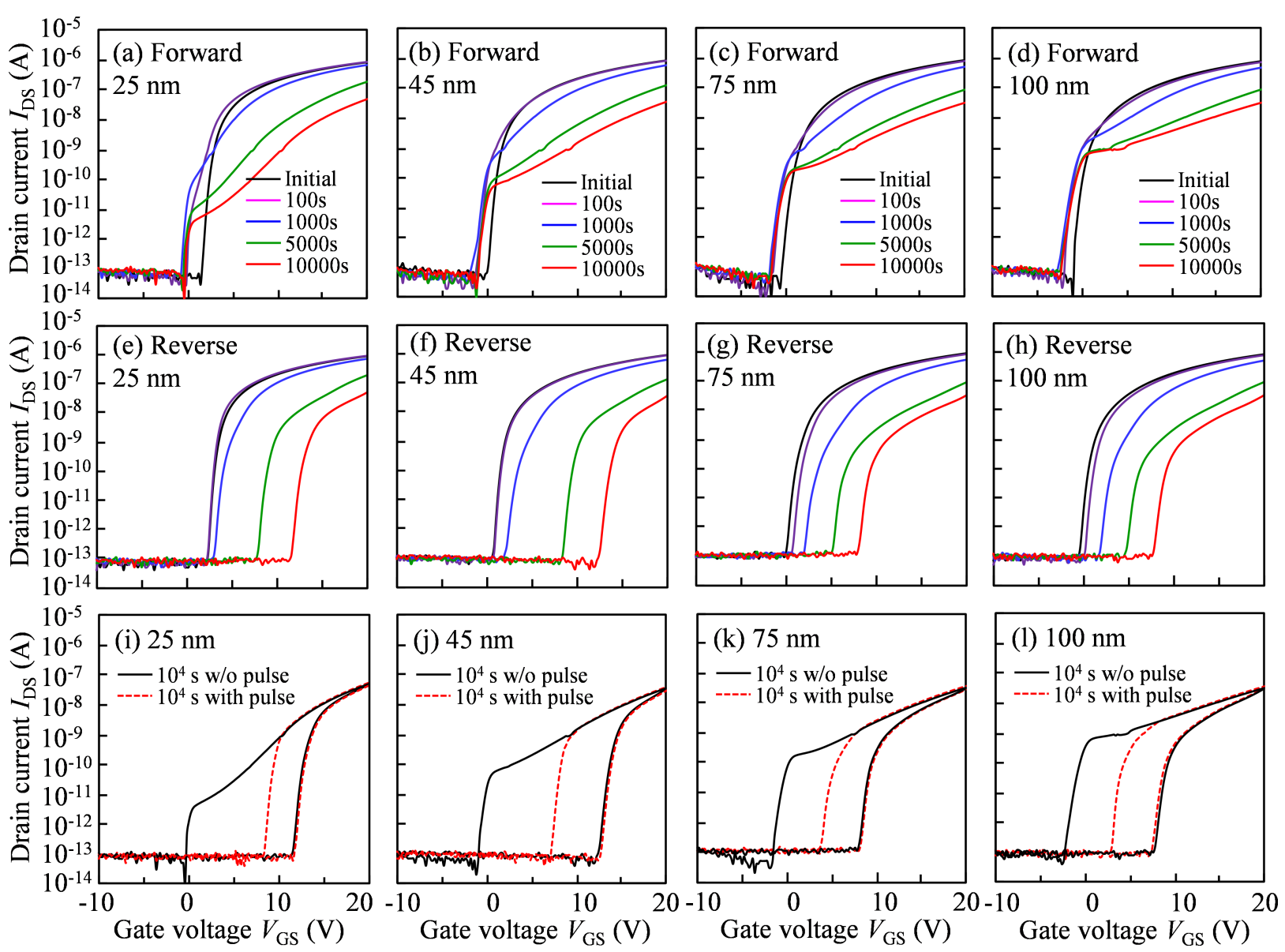

Figure 3: Variation in the transfer characteristics in the forward measurement for a-IGZO TFTs with (a) $T_{\mathrm{IGZO}}=25$, (b) 45 , (c) 75 , and (d) $100 \mathrm{~nm}$, and in the reverse measurement with (e) $T_{I G Z O}=25$, (f) $45,(\mathrm{~g}) 75$, and (h) $100 \mathrm{~nm}$ as a function of the stress duration for $10^{4} \mathrm{~s}$ under $-30 \mathrm{~V} V_{\mathrm{GS}}$ NBIS. Transfer characteristics of a-IGZO TFTs with (i) $T_{I G Z O}=25$, (j) 45 , (k) 75 , and (I) $100 \mathrm{~nm}$ after $10^{4} \mathrm{~s}$ NBIS without and with a gate pulse of $1 \mathrm{~ms}$ width and $10 \mathrm{~V}$ high. 
of $V_{\text {th }}$ without SS degradation is well fitted to the commonly used stretched-exponential equation [28]. The obtained results suggest that electron trapping at the back-channel interface between a-IGZO and etch-stopper layers occurs because a negative gate bias is performed during NBIS.

On the basis of the photoleakage current results, when the photon energy of the light irradiation exceeds $\approx 2.7 \mathrm{eV}$, the photoleakage current of TFTs increases. In this study, a photon energy of $\approx 2.7 \mathrm{eV}$ is set for the incident light. The previous publication indicates that a photon energy of $\approx 2.0$ and $\approx 2.3 \mathrm{eV}$ is required for the transition from $\mathrm{V}_{\mathrm{O}}$ to $\mathrm{V}_{\mathrm{O}}{ }^{+}$and $\mathrm{V}_{\mathrm{O}}{ }^{2+}$, respectively. Moreover, the ionized oxygen vacancies of $\mathrm{V}_{\mathrm{O}}{ }^{+}$and $\mathrm{V}_{\mathrm{O}}{ }^{2+}$ are located near the mid-gap and the bottom of the $E_{\mathrm{C}}$ $[15,29]$, respectively. It is sufficient to excite high-density $\mathrm{V}_{\mathrm{O}}$ defects to $\mathrm{V}_{\mathrm{O}}^{+} / \mathrm{V}_{\mathrm{O}}{ }^{2+}$ and then to generate free electrons to $E_{\mathrm{C}}$. Simultaneously, the electron-hole pairs are photoexcited from $E_{\mathrm{V}}$, which leads to the neutralization between the ionized $\mathrm{V}_{\mathrm{O}}{ }^{+} / \mathrm{V}_{\mathrm{O}}{ }^{2+}$ and the generated electrons, contributing to free holes in $E_{\mathrm{V}}$ [18]. During the NBIS duration with $V_{\mathrm{GS}}=-30 \mathrm{~V}$, a vertical electric field is exerted along the growth direction of the active layer. In general, the electric potential exponentially declines inside the active layer and has a maximum transfer length called the Debye length. In terms of a-IGZO TFT, a Debye length of $\approx 40 \mathrm{~nm}$ is calculated based on a previous publication [30]. In case of a-IGZO TFT with the $T_{\text {IGZO }}=25 \mathrm{~nm}$, the channel layer is totally depleted under the negative $V_{\mathrm{GS}}$ bias since the $T_{\mathrm{IGZO}}$ is less than the Debye length. Therefore, the photoexcited electrons and holes will be respectively accumulated and trapped at the IGZO/etch-stopper and the GI/IGZO interfaces. Meanwhile, the defect states are generated, which originate from the photoexcited $\mathrm{V}_{\mathrm{O}}{ }^{+} / \mathrm{V}_{\mathrm{O}}{ }^{2+}$. In the forward measurement, the transfer curves exhibit a positive shift in the $V_{\mathrm{GS}}$ direction with a hump at the turn-on voltage region when the NBIS duration exceeds $1000 \mathrm{~s}$, which is attributed to the synergistic effects of the generated defect states and the trapped holes at the front-channel interface. After the forward measurement with $V_{\mathrm{GS}}=-10-20 \mathrm{~V}$, the ionized $\mathrm{V}_{\mathrm{O}}{ }^{+} / \mathrm{V}_{\mathrm{O}}{ }^{2+}$ would be gradually neutralized by capturing electrons, and the trapped holes at the front-channel interface are completely de-trapped due to the vertical electric field induced by the positive $V_{\mathrm{GS}}$. Consequently, the abnormal hump observed in the forward measurement disappears in the reverse measurement, suggesting that the donor-like defect states, located near the Fermi level $\left(E_{\mathrm{F}}\right)$ at $V_{\mathrm{GS}}$ of the turn-on voltage, are generated and stabilized in the IGZO layer. It is noted that the trapped electrons at the back-channel interface are hardly de-trapped even when the positive $V_{\mathrm{GS}}$ is applied [17]. As a result, the transfer curves in the reverse measurement exhibit a parallel shift of $10.03 \mathrm{~V}$ without SS degradation in the positive $V_{\mathrm{GS}}$ direction after the NBIS duration of $10^{4} \mathrm{~s}$.

When the $T_{\text {IGZO }}$ is increased to $45 \mathrm{~nm}$, which is close to the Debye length, the whole channel layer is almost depleted under the negative $V_{\mathrm{GS}}$ bias. During the NBIS duration, more electrons and holes are excited and trapped at the back-channel and the front-channel interfaces. Simultaneously, the high-density defect states are generated due to the increase in the photoexcited $\mathrm{V}_{\mathrm{O}}^{+} / \mathrm{V}_{\mathrm{O}}{ }^{2+}$. As a consequence, after the $10^{4} \mathrm{~s}$ NBIS duration, the transfer curves show a significant shift in the positive $V_{\mathrm{GS}}$ direction with a prominent hump for the forward measurement and display a distinct change of $12.46 \mathrm{~V}$ in the positive $V_{\mathrm{GS}}$ direction without SS degradation for the reverse measurement, as shown in Figure $4 \mathrm{a}$. When the $T_{\mathrm{IGZO}}$ is further increased to 75 and $100 \mathrm{~nm}$, which is larger than the Debye length, the electric potential exponentially decreases inside the active layer under $-30 \mathrm{~V} V_{\mathrm{GS}}$ bias. Although some amount of electrons are photoexcited to $E_{\mathrm{C}}$, they are partly accumulated and trapped at the back-channel interface due to the weaker vertical electric field. As a result, the excited hole in $E_{\mathrm{V}}$ and the ionized $\mathrm{V}_{\mathrm{O}}^{+} / \mathrm{V}_{\mathrm{O}}{ }^{2+}$ near $E_{\mathrm{F}}$ at $V_{\mathrm{GS}}$ of the turn-on voltage would be neutralized by the free electrons. Therefore, after the NBIS duration of $10^{4} \mathrm{~s}$, the transfer curves exhibit a small shift in the positive $V_{\mathrm{GS}}$ direction with a weak hump for the forward measure-
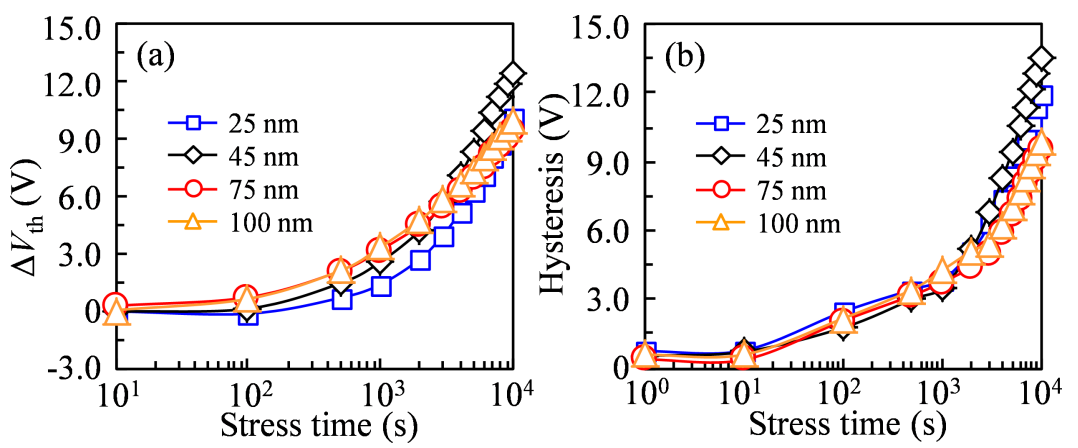

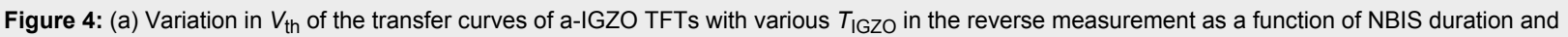
(b) hysteresis of a-IGZO TFTs with various $T_{\mathrm{IGZO}}$ as a function of NBIS duration. 
ment, and show the small shift of 9.45 and $9.96 \mathrm{~V}$ in the positive $V_{\mathrm{GS}}$ direction for the reverse measurement corresponding to $T_{\mathrm{IGZO}}=75$ and $100 \mathrm{~nm}$, respectively.

The combination of the transfer curves in the forward and reverse measurements after the NBIS duration of $10^{4} \mathrm{~s}$ is shown in Figure 4b. The NBIS-induced hysteresis increases remarkably from $11.94 \mathrm{~V}$ for the TFT with $T_{\mathrm{IGZO}}=25 \mathrm{~nm}$ to $13.47 \mathrm{~V}$ for the TFT with $T_{\mathrm{IGZO}}=45 \mathrm{~nm}$, and decreases drastically to 9.54 and $9.93 \mathrm{~V}$ when the $T_{\mathrm{IGZO}}$ further increases to 75 and $100 \mathrm{~nm}$. For further quantitative analysis of the origin of the NBIS-induced hysteresis, a positive gate pulse mode is carried out just after the NBIS duration of $10^{4} \mathrm{~s}$. Based on our previous publication [17], the optimized condition of a positive gate pulse with pulse width of $1 \mathrm{~ms}$ and pulse height of $10 \mathrm{~V}$ is enough to neutralize the ionized $\mathrm{V}_{\mathrm{O}}{ }^{+} / \mathrm{V}_{\mathrm{O}}{ }^{2+}$-induced donor-like defect states while it has no influence on the trapped holes at the front-channel interface, as shown in Figure 3i-1. It is found that the trapped hole-induced hysteresis are $3.41,5.58,4.39$, and $4.62 \mathrm{~V}$ corresponding to the IGZO TFTs with $T_{\mathrm{IGZO}}=25,45$, 75 , and $100 \mathrm{~nm}$, respectively.

To further reveal the mechanism of the NBIS-induced hump and transfer curve shift in a-IGZO TFTs with various $T_{\text {IGZO, }}$ $C-V$ analyses before and after the NBIS duration of $10^{4} \mathrm{~s}$ are measured, as shown in Figure 5. In the initial stage, all $C-V$ curves without distortion are observed, which are in agreement with the initial $I-V$ curves. After the $10^{4} \mathrm{~s}$ NBIS duration, all $C-V$ curves shift in a positive $V_{\mathrm{GS}}$ direction with distortion near the turn-on region. The $C-V$ results suggest that NBIS-induced defect states are uniform in the whole channel layer near $E_{\mathrm{F}}$ at $V_{\mathrm{GS}}$ of the turn-on voltage. In case of a-IGZO TFT with $T_{\mathrm{IGZO}}=25 \mathrm{~nm}$, the $C-V$ curve shifts $11.4 \mathrm{~V}$ in the positive $V_{\mathrm{GS}}$ direction with a hump in the off-state. On the basis of the $C-V$ results, the energy-band diagrams for the IGZO TFTs with various $T_{\text {IGZO }}$ under NBIS are illustrated in Figure 6 . The

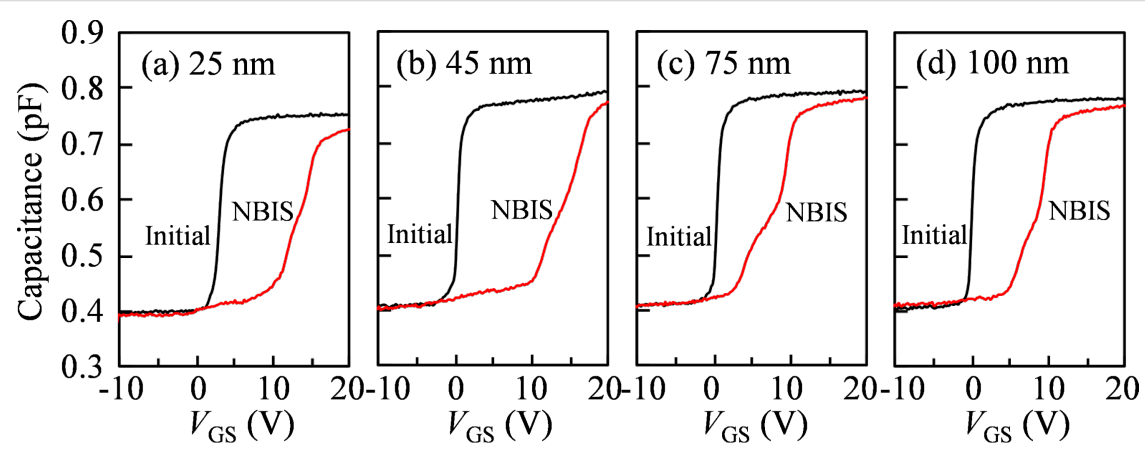

Figure 5: $C-V$ curves before and after the NBIS duration of $10^{4} \mathrm{~s}$ with (a) $T_{\mathrm{IGZO}}=25$, (b) 45 , (c) 75 , and (d) $100 \mathrm{~nm}$, respectively.

(a) $T_{\mathrm{IGZO}}<45 \mathrm{~nm}, \mathrm{NBIS}\left(V_{\mathrm{GS}}=-30 \mathrm{~V}\right)$

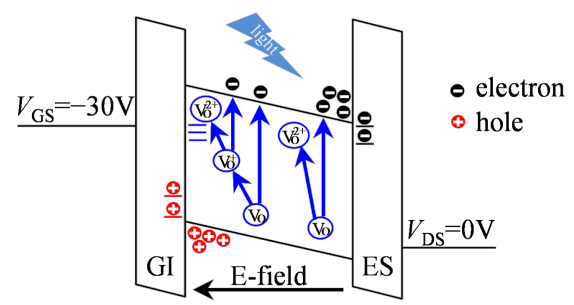

(b) $T_{\mathrm{IGZO}}>45 \mathrm{~nm}$, NBIS $\left(V_{\mathrm{GS}}=-30 \mathrm{~V}\right)$

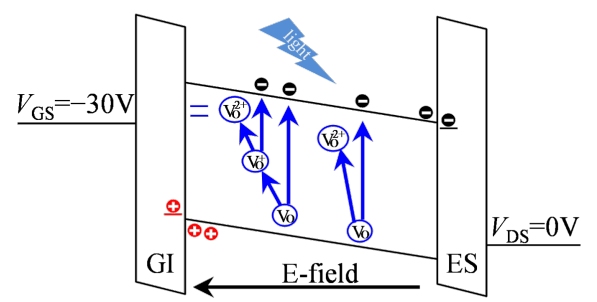

Figure 6: Schematic diagram of NBIS-induced degradation mechanism in a-IGZO TFTs with (a) $T_{\text {IGZO }}<45$ and (b) over the Debye length (75 and $100 \mathrm{~nm})$. 
energy band at the front-channel is remarkably bent upward under the negative $V_{\mathrm{GS}}$ bias when the $T_{\mathrm{IGZO}}$ is less than the Debye length, as shown in Figure 6a. A hump observed at the turn-on region of the $C-V$ curve indicates that the energy level of the generated defect states is located near $E_{\mathrm{F}}$ at $V_{\mathrm{GS}}$ of the turn-on voltage. The positive shift of the $C-V$ curve demonstrates that electrons are trapped at the back-channel interface due to the vertical electric fields in the channel. When the $T_{\text {IGZO }}$ is increased to $45 \mathrm{~nm}$, the $C-V$ curve exhibits a large shift of $15.1 \mathrm{~V}$ in the positive $V_{\mathrm{GS}}$ direction with a distinct hump at the turn-on region. The obtained results suggest that because the $T_{\text {IGZO }}$ is close to the Debye length, the high-density defect states are generated in the whole channel layer and more electrons are photoexcited and trapped at the back-channel interface. When the $T_{\text {IGZO }}$ is further increased to 75 and $100 \mathrm{~nm}$, the $C-V$ curves exhibit smaller shifts of 8.7 and $9.3 \mathrm{~V}$ in the positive $V_{\mathrm{GS}}$ direction with a weaker hump in the off-state compared to the $45 \mathrm{~nm}$-thick channel layer case. The energy band at the front-channel is slightly bent upward as the $T_{\mathrm{IGZO}}$ is much larger than the Debye length, as shown in Figure 6b. The weakened hump near the turn-on region illustrates that the photoexcited $\mathrm{V}_{\mathrm{O}}{ }^{+} / \mathrm{V}_{\mathrm{O}}{ }^{2+}$ would be neutralized by the free electrons in $E_{\mathrm{C}}$, contributing to the low-density defect states near $E_{\mathrm{F}}$ at $V_{\mathrm{GS}}$ of turn-on voltage. The small shift of the $C-V$ curves demonstrates that the fewer electrons are accumulated and trapped at the back-channel interface. The obtained $C-V$ results are correlated with the results of the $I-V$ measurements.

On the basis of the above discussion, it is demonstrated that the $T_{\text {IGZO }}$ is one of the critical parameters to modify the electrical properties of the device. Besides the active layer thickness, the intrinsic characteristics of a-IGZO and the front- and backchannel interfaces of the TFT also play a vital role for the highperformance devices. Moreover, to reduce the density of oxygen vacancies in the bulk of the IGZO for the enhancement of electrical properties and stress stability of the TFTs, the following two aspects should be mainly considered: (i) oxidizing the densities of the defect state of oxide semiconductors to suppress charge trapping, for example by oxygen annealing and $\mathrm{N}_{2} \mathrm{O}$ plasma treatment [31]; and (ii) inactivating the defects in the semiconductor by means of introducing new elements to form stable chemical bonds with the defects, for example by fluoride ion implantation and nitrogen annealing [32,33].

\section{Conclusion}

The impact of the $T_{\text {IGZO }}$ on the photoleakage current and the NBIS-induced instability in a-IGZO TFTs were systematically investigated. It was found that when the photon energy of the light irradiation exceeds $\approx 2.7 \mathrm{eV}$, the photoleakage current increases in all TFTs irrespective of the $T_{\text {IGZO }}$ due to the high- density electron traps existing at an $\left(E_{\mathrm{C}}-E\right)$ of $\approx 2.7 \mathrm{eV}$. Because the total amount of trapped electrons increases with increasing $T_{\text {IGZO }}$, the photoleakage current gradually increases with increasing $T_{\text {IGZO }}$. On the basis of the photoleakage current results, the influence of the $T_{\text {IGZO }}$ on NBIS with a photon energy of $\approx 2.7 \mathrm{eV}$ in a-IGZO TFTs is clarified by the $I-V$ and $C-V$ measurements. In addition, the NBIS-induced hysteresis is quantitatively evaluated through a positive gate pulse mode, contributing to the separation of the trapped holes at the frontchannel interface and the generation of donor-like defect states in a-IGZO layer. The obtained $I-V$ and $C-V$ results indicate that when the $T_{\text {IGZO }}$ is close to the Debye length, the trapped holes at the front-channel interface, the trapped electrons at the backchannel interface, and the generated donor-like defect states in a-IGZO are distinctly prominent during NBIS. This study suggests that to improve the reliability of oxide TFTs under light irradiation and gate bias stresses, the quality of the active layer and interface engineering should be taken into account.

\section{Acknowledgements}

The authors acknowledge all support from the National Key Research and Development Program of China (2016YFA0202403), the National Nature Science Foundation of China (61674098, 91733301), the Natural Science Foundation of Shaanxi Provincial Department of Education (2017KW023, 2017JM6020), the Fundamental Research Funds for the Central Universities (GK201603053, GK201702003, GK201601010), the Changjiang Scholar and the Innovative Research Team (IRT_14R33), the 111 Project (B14041), and the Chinese National 1000-talent-plan program (Grant No. 111001034). This work was partly supported by JSPS KAKENHI Grant Number 16K06309.

\section{ORCID ${ }^{\circledR}$ iDs}

Dapeng Wang - https://orcid.org/0000-0001-9897-0627

Mamoru Furuta - https://orcid.org/0000-0003-1685-3246

\section{References}

1. Nomura, K.; Ohta, H.; Takagi, A.; Kamiya, T.; Hirano, M.; Hosono, H. Nature 2004, 432, 488-492. doi:10.1038/nature03090

2. Zhang, J.; Yang, J.; Li, Y.; Wilson, J.; Ma, X.; Xin, Q.; Song, A. Materials 2017, 10, 319. doi:10.3390/ma10030319

3. Hirao, T.; Furuta, M.; Hiramatsu, T.; Matsuda, T.; Li, C.; Furuta, H.; Hokari, H.; Yoshida, M.; Ishii, H.; Kakegawa, M. IEEE Trans. Electron Devices 2008, 55, 3136-3142. doi:10.1109/ted.2008.2003330

4. Tsai, C.-T.; Chang, T.-C.; Chen, S.-C.; Lo, I.; Tsao, S.-W.; Hung, M.-C.; Chang, J.-J.; Wu, C.-Y.; Huang, C.-Y. Appl. Phys. Lett. 2010, 96, 242105. doi:10.1063/1.3453870

5. Kim, K. S.; Ahn, C. H.; Kang, W. J.; Cho, S. W.; Jung, S. H.; Yoon, D. H.; Cho, H. K. Materials 2017, 10, 530. doi:10.3390/ma10050530 
6. Liu, A.; Liu, G.; Zhu, C.; Zhu, H.; Fortunato, E.; Martins, R.; Shan, F. Adv. Electron. Mater. 2016, 2, 1600140. doi:10.1002/aelm.201600140

7. Kim, Y. G.; Kim, T.; Avis, C.; Lee, S.-H.; Jang, J. IEEE Trans. Electron Devices 2016, 63, 1078-1084. doi:10.1109/ted.2016.2518703

8. Liu, X.; Jiang, L.; Zou, X.; Xiao, X.; Guo, S.; Jiang, C.; Liu, X.; Fan, Z.; Hu, W.; Chen, X.; Lu, W.; Hu, W.; Liao, L. Adv. Mater. 2014, 26, 2919-2924. doi:10.1002/adma.201305073

9. Shimakawa, S.-i.; Kamada, Y.; Kawaharamura, T.; Wang, D.; Li, C.; Fujita, S.; Hirao, T.; Furuta, M. Jpn. J. Appl. Phys. 2012, 51, 03 CB04. doi:10.7567/jjap.51.03cb04

10. Shimakawa, S.-i.; Wang, D.; Furuta, M. Jpn. J. Appl. Phys. 2012, 51, 108003. doi:10.7567/jjap.51.108003

11. Chang, Y.-G.; Moon, T.-W.; Kim, D.-H.; Lee, H. S.; Kim, J. H.; Park, K.-s.; Kim, C.-D.; Im, S. IEEE Electron Device Lett. 2011, 32, 1704-1706. doi:10.1109/led.2011.2167736

12. Oh, H.; Yoon, S.-M.; Ryu, M. K.; Hwang, C.-S.; Yang, S.; Park, S.-H. K. Appl. Phys. Lett. 2011, 98, 033504. doi:10.1063/1.3540500

13. Kim, B.; Chong, E.; Hyung Kim, D.; Woo Jeon, Y.; Hwan Kim, D.; Yeol Lee, S. Appl. Phys. Lett. 2011, 99, 062108. doi:10.1063/1.3615304

14. Chen, T. C.; Chang, T. C.; Hsieh, T. Y.; Tsai, C. T.; Chen, S. C.; Lin, C. S.; Jian, F. Y.; Tsai, M. Y. Thin Solid Films 2011, 520 1422-1426. doi:10.1016/j.tsf.2011.09.002

15. Oh, H.; Yoon, S.-M.; Ryu, M. K.; Hwang, C.-S.; Yang, S.; Park, S.-H. K. Appl. Phys. Lett. 2010, 97, 183502. doi:10.1063/1.3510471

16. Huang, S.-Y.; Chang, T.-C.; Lin, L.-W.; Yang, M.-C.; Chen, M.-C.; Jhu, J.-C.; Jian, F.-Y. Appl. Phys. Lett. 2012, 100, 222901. doi:10.1063/1.4722787

17. Wang, D.; Hung, M. P.; Jiang, J.; Toda, T.; Furuta, M. ACS Appl. Mater. Interfaces 2014, 6, 5713-5718. doi:10.1021/am500300g

18. Hung, M. P.; Wang, D.; Jiang, J.; Furuta, M. ECS Solid State Lett. 2014, 3, Q13-Q16. doi:10.1149/2.010403ssl

19. Park, H.-W.; Park, K.; Kwon, J.-Y.; Choi, D.; Chung, K.-B. IEEE Trans. Electron Devices 2017, 64, 159-163. doi:10.1109/ted.2016.2630043

20. Park, J.; Kim, Y. S.; Kim, J. H.; Park, K.; Park, Y. C.; Kim, H.-S. J. Alloys Compd. 2016, 688, 666-671. doi:10.1016/j.jallcom.2016.07.245

21. Yang, Z.; Yang, J.; Meng, T.; Qu, M.; Zhang, Q. Mater. Lett. 2016, 166, 46-50. doi:10.1016/j.matlet.2015.12.029

22. Avis, C.; Hwang, H. R.; Jang, J. ACS Appl. Mater. Interfaces 2014, 6, 10941-10945. doi:10.1021/am501153w

23. Jeong, J.; Hong, Y. IEEE Trans. Electron Devices 2012, 59, 710-714. doi:10.1109/ted.2011.2180908

24. Wang, D.; Hung, M. P.; Jiang, J.; Toda, T.; Li, C.; Furuta, M. Jpn. J. Appl. Phys. 2014, 53, 03CC01. doi:10.7567/jjap.53.03cc01

25. Wang, D.; Zhao, W.; Li, H.; Furuta, M. Materials 2018, 11, 559. doi:10.3390/ma11040559

26. Nomura, K.; Kamiya, T.; Yanagi, H.; Ikenaga, E.; Yang, K.; Kobayashi, K.; Hirano, M.; Hosono, H. Appl. Phys. Lett. 2008, 92, 202117. doi:10.1063/1.2927306

27. Kamiya, T.; Nomura, K.; Hosono, H. J. Disp. Technol. 2009, 5, 468-483. doi:10.1109/jdt.2009.2034559

28. Libsch, F. R.; Kanicki, J. Appl. Phys. Lett. 1993, 62, 1286-1288. doi:10.1063/1.108709

29. Janotti, A.; Van de Walle, C. G. Appl. Phys. Lett. 2005, 87, 122102. doi:10.1063/1.2053360
30. Li, Y.; Pei, Y. L.; Hu, R. Q.; Chen, Z. M.; Zhao, Y.; Shen, Z.; Fan, B. F.; Liang, J.; Wang, G. Curr. Appl. Phys. 2014, 14, 941-945. doi:10.1016/j.cap.2014.04.011

31. Chang, G.-W.; Chang, T.-C.; Jhu, J.-C.; Tsai, T.-M.; Syu, Y.-E.; Chang, K.-C.; Tai, Y.-H.; Jian, F.-Y.; Hung, Y.-C. Appl. Phys. Lett. 2012, 100, 182103. doi:10.1063/1.4709417

32. Wang, D.; Jiang, J.; Furuta, M. J. Disp. Technol. 2016, 12, $258-262$. doi:10.1109/jdt.2015.2472981

33. Yoon, S.; Tak, Y. J.; Yoon, D. H.; Choi, U. H.; Park, J.-S.; Ahn, B. D.; Kim, H. J. ACS Appl. Mater. Interfaces 2014, 6, 13496-13501. doi:10.1021/am502571w

\section{License and Terms}

This is an Open Access article under the terms of the Creative Commons Attribution License

(http://creativecommons.org/licenses/by/4.0). Please note that the reuse, redistribution and reproduction in particular requires that the authors and source are credited.

The license is subject to the Beilstein Journal of Nanotechnology terms and conditions: (https://www.beilstein-journals.org/bjnano)

The definitive version of this article is the electronic one which can be found at: doi:10.3762/bjnano.9.239 